\title{
Review on Energy Efficient Routing Protocols in WSNs
}

\author{
Paramjit Singh $^{1}$, Tejinderdeep Singh ${ }^{2}$ \\ M. Tech Student, Computer Science, Global Institute of Management and Emerging Technology, \\ PTU Jalandhar, Punjab ${ }^{1}$ \\ Asst. Prof, Computer Science Department, Global Institute of Management and Emerging Technology, \\ PTU Jalandhar, Punjab ${ }^{2}$
}

\begin{abstract}
Wireless Sensor Network is a highly distributed network of small and lightweight sensing nodes which are deployed in a large number at multiple locations. These networks are helpful in monitoring the system or environment. These sensor nodes performs sensing, processing and then communicating. Now a day's these networks are applied in various fields like military, defence, forest fire, medical, crisis management etc. In this paper it has been discussed about sensor network architecture and major factors affecting the wireless sensor networks. Routing is a major challenge faced by wireless sensor networks due to the dynamic nature of WSNs. Since energy efficient routing protocols are of major concern in the field of wireless sensor network. Therefore in further sections of this paper it has presented a brief review of different energy efficient routing protocols proposed for wireless sensor networks.
\end{abstract}

Keywords: Clustering, Energy efficient routing, LEACH, Sensor Nodes, Wireless Sensor Networks, WSNs.

\section{INTRODUCTION}

A wireless sensor network is a cooperative network which consists of an organized collection of nodes. The nodes communicate wirelessly and can self-organize themselves after being deployed in an ad hoc fashion. Systems of multiple 1000s of nodes are anticipated. Such systems can revolutionize our life \& works. Recently, wireless sensor networks are being deployed very rapidly. It is not unreasonable to expect that in coming future the world will be covered with wireless sensor networks with Internet accessibility. This can be considered as the Internet transforming into a physical network. This new technology is overexciting with unexpected potential for number of new application areas including environmental, medical, military, transportation, entertainment, crisis management, defense, and many more. However wireless sensor network is a resource constraint if we talk about energy, power computation, memory and limited communication capabilities. All sensing nodes in the wireless sensor network interact with each other directly or by intermediate sensor nodes. A sensor node that produces data based on its sensing observations and transmit sensed data packet to the base station (sink). This process basically involves direct transmission because the base station may locate very far away from the location of sensor nodes. More energy is required to transmit data over long distances so that a robust technique is to have fewer nodes send data to the base station. These nodes called head nodes and perform data aggregation in wireless sensor network. A Careful management of resources is needed to increase the lifetime of the wireless sensor network. Routing is main challenge faced by wireless sensor network. Due to dynamic nature of WSNs; routing is complex. Quality of routing protocols depends upon the amount of data (actual data signal) successfully received by Base station from sensors nodes deployed in the network region.

The organization of the paper is as follows: section 2 discusses different types of sensor network architecture; section 3 discusses about different factors affecting the wireless sensor networks; section 4 contains a brief review of different energy efficient routing protocols proposed for wireless sensor networks; section 5 concludes the paper.

\section{NETWORK ARCHITECTURE}

Sensor network architecture can be either of following:

1. Layered Architecture

2. Clustered Architecture

\section{Layered Architecture}

In this architecture there is a single powerful base station. And there is layer of sensor nodes around base station. For any layer $\mathrm{N}$, all nodes are $\mathrm{N}$-hop away from base station. 


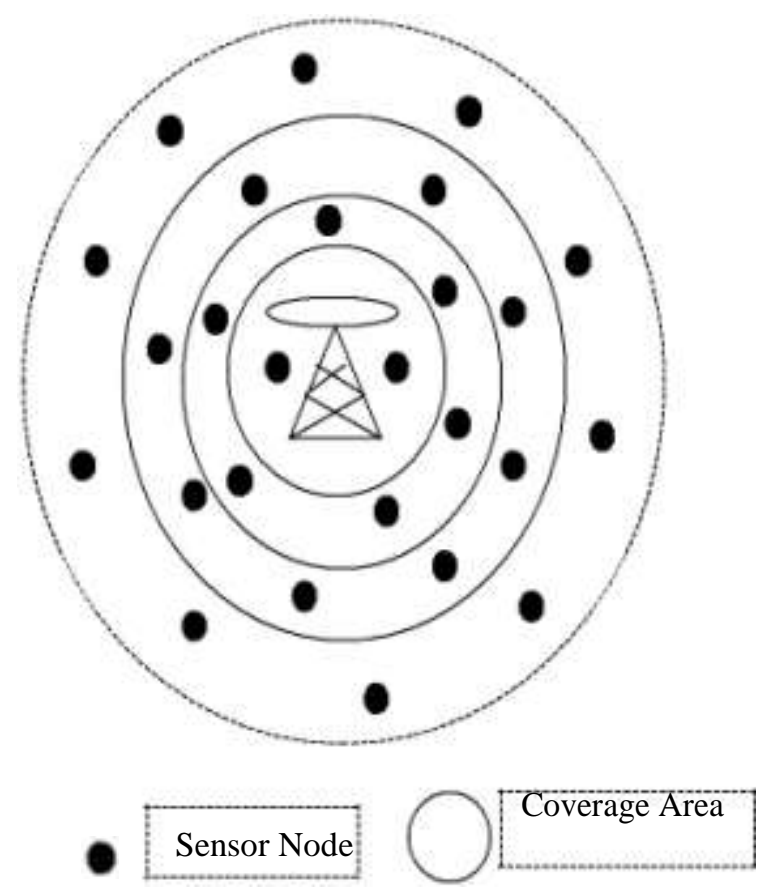

Fig 2.1 Layered WSN Architecture Clustered Architecture

In this architecture sensor nodes are organized into clusters. Each cluster is addressed by a cluster head. Only cluster head sends messages to the base station.

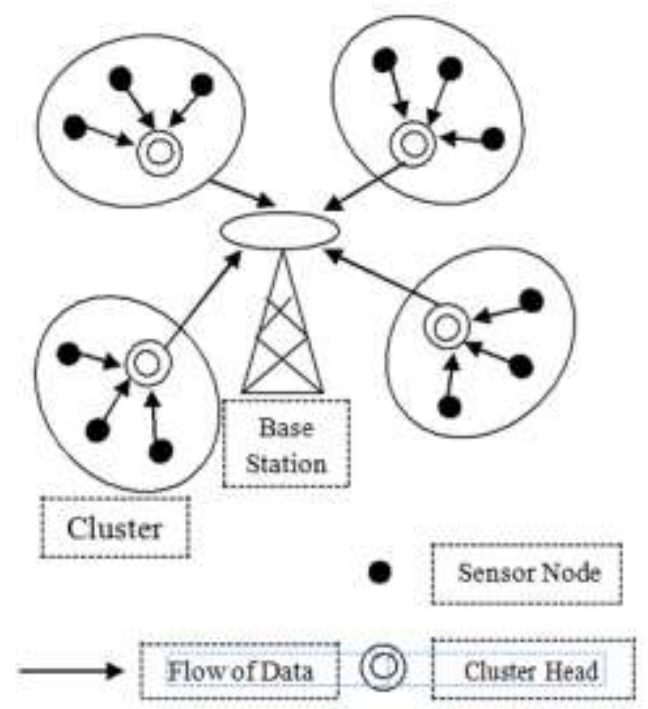

Fig 2.2 Clustered WSN Architecture

\section{FACTORS AFFECTING WSNS}

Following are some of the major factors which affect the wireless sensor networks.

\section{Network Characteristics}

Network formed by the tiny sensor nodes can be homogenous or heterogeneous. Homogenous WSNs is composed of same level of devices \& comprising the same hardware capabilities using the same policy. The functionality of a homogenous network serves the purpose of gathering the sensed data and sending it to a central location which is beneficial for real time \& environment application. Whereas heterogeneous WSNs use various ranges of different devices which are able t cooperate in order to achieve a global goal by combining the individual capabilities of the nodes. 


\section{Energy Consumption}

Energy is an important attribute of WSNs because each sensor nodes have a limited amount of battery lifetime. Moreover energy is consumed in following things:

i. Each sensor node consumes energy for sensing communicating \& cluster formation.

ii. Each Cluster Head spends energy in different domains for receiving aggregating \& transmitting the signals.

iii. Energy is also invested in the process to listen the channel for any incoming packets.

\section{Routing}

Routing is the main challenge faced by wireless sensor networks because of dynamic nature of WSNs. Quality of routing protocols depends upon the amount of data i.e. actual data signal successfully received by base station from sensor nodes deployed in the network region. Routing protocols may be either:

i. $\quad$ Flat Routing protocols

ii. Hierarchical routing protocols

iii. Location based routing protocols.

Routing can be single hop or multi hop. Multi hop routing is used because when data is sent directly in between Cluster Head \& Base Station then due to increased distance can lead to the path loss exponent due to multipath fading.

\section{Scalability}

Scalability of routing protocols used in wireless sensor networks (WSNs) is a critical issue due to the extremely high node numbers and relatively high node density. A good routing protocol should be scalable and adaptive to the runtime changes in the network topology. Hence protocols must perform well as the network grows larger or as the workload increases. [13]

\section{Fault Tolerance}

Fault tolerance is one of the very important among several challenges in these networks. Fault tolerance improves wireless sensor networks (WSN) with reliable collection and dissemination of data with preserving limited resources in sensor nodes, especially power. However data redundancy achieves the goal of fault tolerance in the data-centered network infrastructure of WSNpsilas, it also incurs security concerns by making data available in several locations. More attention needs to be paid when WSNpsilas are deployed in hostile environments where sensor nodes are easy to be captured for deleterious use by an adversary. In this context, cryptographic keys are of low efficiency for protecting data not involved in communication [14].

\section{Reliability}

Reliable transmission of data is one of the major characteristic of most of the existing transport protocols in traditional networks or WSNs. Reliability evaluation of wireless sensor networks (WSN) is a critical step in WSN design. Reliability is defined as the amount of packets received by the sink to the total number $f$ packets generated by the end nodes [9].

\section{Timeliness}

Timeliness refers to obtaining the collected data from the WSN in a timely manner such that control actions can be taken depending on the status of the system [9]. Wide spread use of wireless sensor networks (WSNs) in sensitive applications shows the importance of Quality of service (QoS) in these networks. Timeliness is also one of the QoS parameters which have big importance in real time applications such as earthquake detection. Multiple routing protocols have been proposed for WSNs which provide QoS support in this domain. Most of these protocols use onehop neighborhood information to perform routing decisions. Timeliness may be improved by using multi-hop neighborhood nodes information which may add extra control overhead and decreases network lifetime. In other words, the choice of two-hop neighborhood information is a tradeoff between timeliness and control overhead [15].

\section{Mobility}

Among the many ways of improving the performance of a wireless sensor network (WSN) in terms of crucial metrics such as its lifetime and data latency, exploiting the mobility of some of the network components has been recently observed to be among the most promising. For example we can reduce communication cost by sink mobility. Sink moves randomly by changing its position after every round. This uniforms energy consumption and increases network lifetime [3].

\section{Data Aggregation}

A fundamental challenge in the design of wireless sensor networks (WSNs) is to maximize their lifetimes. Data Aggregation has emerged as a promising approach in WSNs in order to reduce the number of transmissions of sensor 
nodes, and hence decreasing the overall energy consumption in the network. Data aggregation is affected by many factors, such as the placement of aggregation nodes, the aggregation function, and the density of sensor nodes in the network. The determination of an optimal selection of aggregation nodes is thus extremely important [16].

\section{Delay}

Wireless sensor networks prove useful in many delay-sensitive applications, e.g., emergency response and plant automation. In such networks, delay measurement is of key importance for a number of reasons, e.g., real-time control and abnormal delay detection. There are mainly four factors that affect the end-to-end delay in WSNs [8]:

i. Transmission delay. It is limited by the link bandwidth

ii. Competition of the radio channel. Especially under a contention based MAC, a packet has to compete for the access of the channel and wait for transmission until the channel is idle.

iii. Queuing delay. A large queue will seriously delay packets;

iv. Path length. Generally, the more hops a packet travels, the large propagation delay it will suffer.

\section{ENERGY EFFICIENT ROUTING PROTOCOLS IN WSNs}

Network topology and routing algorithm have a great impact on the performance of WSNs. Recent researches carried out in the field of cluster-based routing algorithms, have investigated the algorithms from different perspectives.

Among the previously proposed algorithms, the LEACH algorithm [18] has significantly increased the WSN lifetime in comparison with previous non-clustered routing algorithms. It uses the $\mathrm{CH}$ nodes as routers to the BS and all data processing e.g., data fusion and aggregation are done locally to the clusters. In each round of LEACH algorithm, the initialization and stabilization phases are performed. During the initialization phase, $\mathrm{CH}$ nodes are selected among sensor nodes; after that, other nodes are joined to their nearest $\mathrm{CH}$ node in terms of distance and thereby clusters are formed. In the process of $\mathrm{CH}$ nodes selection, each sensor node will generate a random number between 0 and 1 . If the random number generated is less than the $T(n)$ threshold, where $T(n)=p /(1-p *(r \bmod 1 / p))$, then the node is selectedas $\mathrm{CH}$ node. In this equation, $\mathrm{p}$ is the probability of selecting nodes as a $\mathrm{CH}$ node in the sensor populations; $r$ is the current round number; and $(\mathrm{r}$ mod $(1 / p))$ is on behalf of the number of nodes which was elected as $\mathrm{CH}$ node in the round $\mathrm{r}$. The sensor nodes within each cluster transmit the received data from the environment to their $\mathrm{CH}$ nodes in the slot assigned to them by $\mathrm{CH}$ node. In the stability phase, the $\mathrm{CH}$ nodes transmit gathered information to the BS. Because of the hierarchical nature of the LEACH algorithm, the operation of the route selection is simple and the routing of data, does not require much information. Furthermore, according to the random mechanism of $\mathrm{CH}$ node selection, the opportunities of all sensor nodes to be $\mathrm{CH}$ node are equal. However, the $\mathrm{LEACH}$ algorithm also has some disadvantages; such as the energy of $\mathrm{CH}$ nodes which are away from the BS has been quickly evacuated. Therefore, the development of network under the LEACH algorithm is not feasible. The other is the LEACH algorithm has not consider the sensor node position factor, so it does not makes the appropriate $\mathrm{CH}$ nodes distribution. Energy factor in the LEACH algorithm are not cconsidered in the $\mathrm{CH}$ nodes selection. Therefore, the probability of selecting sensor nodes with different energy levels is equal.

Lots many works have been done by the authors to optimize or avoid the various issues associated with LEACH. Brief introduction to some are as follows [6]:

LEACH-E (Energy Low Energy Adaptive Clustering Hierarchy) involves a cluster head selection algorithm which has non-uniform starting energy level among the sensors having global information about the other sensors. In order to minimize the total energy consumption the required number of cluster heads has to scale as the square root of the total number of sensor nodes and this can be determined by Leach-E. By considering the residual energy of the sensor node as the main factor, it decides whether the sensor nodes can be turned into the cluster head or not in the next-round.

LEACH-B (Balanced Low Energy Adaptive Clustering Hierarchy) uses the decentralized algorithms of cluster formation where each sensor node only knows about its own position and the final receiver and does not know about the position of all the sensor nodes. Leach-B involves the following techniques Cluster head selection algorithm, Cluster formation and data transmission with multiple accesses. By evaluating the energy dissipated in the path between final receiver and itself, each of the sensor nodes chooses its cluster head. Efficiency of Leach-B is better than Leach.

In LEACH-A (Advanced Low Energy Adaptive Clustering Hierarchy) method, the head node consumes more energy than others. Hence the energy saving and reliable data transfer is improved LEACH-A. In this, the data is processed using mobile agent technique based on Leach. Advanced Leach, a heterogeneous energy protocol is proposed for the purpose of decreasing the node's failure probability and for prolonging the time interval before the death of the first node which can be referred to as stability period. 
LEACH-M (Mobile - Low Energy Adaptive Clustering Hierarchy) is proposed to mitigate the mobility issue present in LEACH. It involves the mobility of non-cluster head nodes and cluster head during the setup and steady state phase. The nodes in Leach-Mare assumed to be homogeneous and have their location information through GPS. The cluster head can be selected based on the minimum mobility and lowest attenuation mode. The selected cluster heads then broadcasts their status to all nodes in transmission range.

In LEACH-S (SOLAR AWARE CENTRALIZED LEACH), the base station selects the cluster head with the help of improved central control algorithm. Base station selects solar powered nodes having maximum residual energy. In Leach-S, the solar status is transmitted by the nodes to the base station along with the energy and the nodes with higher energy are selected as the cluster head. When the number of solar-aware nodes is increased, the performance of sensor network is also increased. The sun duration increases the lifetime of the sensor network. The cluster head handover takes place if the sun duration is smaller.

In LEACH-S (SOLAR AWARE DISTRIBUTED LEACH), the solar driven nodes are given choosing the preference of cluster head probability of solar driven nodes which is higher than the battery driven nodes. All the descendants of LEACH routing protocols share some common traits. Still there is a need of modification in existing LEACH protocols in order to use the slots corresponding to nodes that do not have data to send a scheduled slot.

V LEACH (VICE LEACH) protocol is similar to LEACH protocol. According to LEACH protocol CH is the node which is responsible for sending and receiving of data to base station. Therefore energy dissipation is most in $\mathrm{CH}$ rather than the member nodes. [22][23] This could lead to death of a $\mathrm{CH}$ and network to stop working. To prevent this $\mathrm{V}$ LEACH protocol consists of a vice $\mathrm{CH}$ along with a $\mathrm{CH}$. In such case when the $\mathrm{CH}$ node dies, vice $\mathrm{CH}$ node will take over the role of $\mathrm{CH}$ so the network performance is not affected.

TL LEACH (TWO LEVEL LEACH) - This is two level leach protocol used to reduce the energy dissipation by cluster head situated far away from base station. As we know transmission requires energy and if it is far away it will require more energy and $\mathrm{CH}$ will die soon. So in this protocol the processed data is transmitted to other $\mathrm{CH}$ which is located in between the path of $\mathrm{CH}$ and Base station. Thus it reduces the burden of transmitting data through single $\mathrm{CH}$. It works as a relay station and helps in reducing energy consumption of $\mathrm{CH}$. [23].

MULTI HOP LEACH is similar to LEACH protocol but communication path from cluster head to base station is converted from single hop to multi hop.[21] Data is transmitted to base station through various cluster head .It transfers data from one cluster head to corresponding cluster head which is nearest to base station. It selects optimal path with lesser number of hop count between first cluster head and base station. In this type of LEACH protocol energy is efficiently used by network because far away cluster head require low energy for sending data than nearer cluster head. This reduces the energy dissipation of single $\mathrm{CH}$ if it is situated far away from base station. Thus it increases the life span of network.

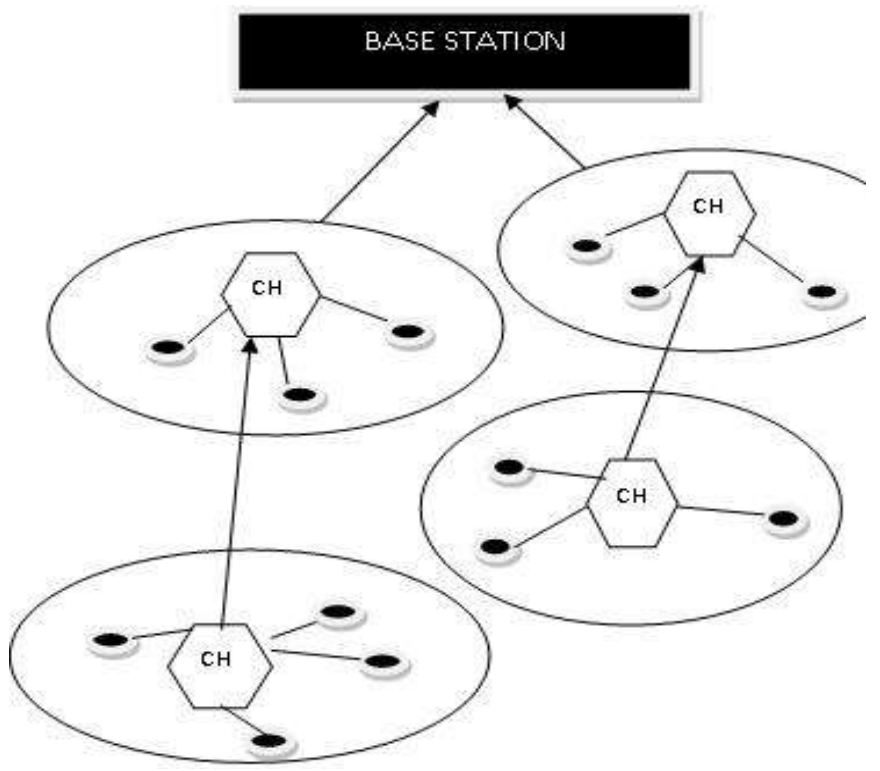

Figure 4.1- Clustering in MULTI HOP LEACH protocol 
I-LEACH (Improved Low Energy Adaptive Clustering Hierarchy) [7] considers sensor nodes current energy, sensor nodes position, and the number of neighbours of sensor nodes in the selection of $\mathrm{CH}$ nodes. By selection of sensor nodes that have more remaining energy, and also have the relatively little distance for being $\mathrm{CH}$ node, as well as by taking into account the distance of $\mathrm{CH}$ nodes from the $\mathrm{BS}$ factor in the cluster formation phase.

Op-LEACH (Optimized Low Energy Adaptive Clustering Hierarchy) [6] utilizes the slots belonging to the node having no data to send. This method turns free slots into useful slots without making any changes in the TDMA schedule. This will reduce the waiting time for sensor nodes because now sensor nodes can get more than one slot per frame. It will reduce the data transmission delay and increase throughput of the network.

PEGASIS algorithm [19], an approximate optimal algorithm based on chain structure of sensor nodes that improved LEACH algorithm; in this algorithm the operations of chain implement and chain control can be done by both the BS and the sensor nodes. Sensor nodes on the chain are merely connected with their close neighbors and receive from and transfer to them and sensor nodes take turns transmitting to the BS; thus the average of spent energy by each node per round has reduced. But under the PEGASIS algorithm, all nodes need to have a general knowledge of the network to form chains and greedy algorithms are used. This problem increases PEGASIS algorithm spent cost and makes it difficult to implement of it.

HEED algorithm [17], expands LEACH algorithm scheme by applying a combination of factors like remaining energy and number of neighbors. In HEED algorithm, unlike LEACH, CH nodes are well distributed in the network. But, this algorithm does not consider any assumptions about the sensor node capabilities, such as geographical position. LEACH-C algorithm, forms better clusters via the dispersing of $\mathrm{CH}$ nodes within the network. Besides, during the initialization phase of LEACH-C algorithm, each sensor node sends information about its current position and residual energy level to the BS. Therefore the BS manages the clustering process having a more efficient knowledge of the network.

TEEN (Threshold sensitive energy efficient Sensor network protocol).It is one of the hierarchical clustering protocol in which hierarchy of sensor nodes is present. In this protocol data is accumulated from sensor nodes and transmitted from cluster head of first level to cluster head of next level and so on until it reaches to the base station. TEEN executes its function on the basis of a threshold value. It is the best energy efficient protocol as it implies a constraint on when the sensor should transmit the data thus reducing number of transmissions.

APTEEN (Adaptive threshold sensitive energy efficient sensor network protocol).It is an enhancement of TEEN protocol in order to overcome its drawbacks. It uses the same concept of TEEN to reduce energy dissipation. This protocol provides a time critical information as well as constant transmission of sensed data to user. It works on the combination rule from both the LEACH and TEEN protocol. Its efficiency is between the two protocols as it performs the function of both the protocol.

\section{CONCLUSION}

In this review paper a brief introduction about wireless sensor networks is given. It has also discussed about sensor network architecture. The main concern of this paper was to present a review on major factors affecting the WSNs. Energy is an important concern in wireless sensor networks due to its limited lifetime capabilities so here it has also presented a survey on different energy efficient routing protocols in the field WSNs.

A comparison between major clustering protocol is concluded in the below mention table.

Table 1: Comparison of major clustering protocols on various parameters [20]

\begin{tabular}{|c|c|c|c|c|c|c|}
\hline Protocol & Scalability & $\begin{array}{c}\text { Cluster } \\
\text { Stability }\end{array}$ & $\begin{array}{c}\text { Energy } \\
\text { Efficiency }\end{array}$ & $\begin{array}{c}\text { Load } \\
\text { Balancing }\end{array}$ & $\begin{array}{c}\text { Algorithm } \\
\text { Complexity }\end{array}$ & Delivery Delay \\
\hline LEACH & Very Low & Moderate & Very Low & Moderate & Low & Very Small \\
\hline HEED & Moderate & High & Moderate & Moderate & Moderate & Moderate \\
\hline PEGASIS & Very Low & Low & Very High & Moderate & High & Very Large \\
\hline TEEN & Low & Very Low & Moderate & Good & High & Small \\
\hline APTEEN & Low & Low & Low & Moderate & Very High & Small \\
\hline
\end{tabular}




\section{REFERENCES}

[1] Rani, S.; Malhotra, J.; Talwar, R., "Energy efficient protocol for densely deployed homogeneous network," Issues and Challenges in Intelligent Computing Techniques (ICICT), 2014 International Conference on ,vol., no., pp.292,298, 7-8 Feb. 2014.

[2] Munjal, Amit; Tripathi, Rajiv Kr.; Singh, Y.N., "Balancing energy consumption using cluster based approach in wireless sensor network," Communications(NCC), 2014 Twentieth National Conference on, vol.,no., pp.1,5, Feb. 28 2014-March 22014.

[3] Deepali; Padmavati, "Improved energy efficiency semi static routing algorithm using sink mobility for WSNs," Engineering and Computational Sciences (RAECS), 2014 Recent Advances in , vol., no., pp.1,5, 6-8 March 2014

[4] Lehsaini, Mohamed; Guyennet, Herve, "Checkpoint-based Fault-tolerance for LEACH Protocol," NewTechnologies, Mobility and Security (NTMS), 2014 6th International Conference on , vol., no., pp.1,4, March 302014-April 22014

[5] Rahayu, T.M.; Sang-Gon Lee; Hoon-Jae Lee, "Survey on LEACH-based security protocols," Advanced Communication Technology (ICACT), 2014 16th International Conference on , vol., no., pp.304,309, 16-19 Feb. 2014

[6] Gambhir, S.; Fatima, N., "Op-LEACH: An Optimized LEACH Method for Busty Traffic in WSNs," AdvancedComputing \& Communication Technologies (ACCT), 2014 Fourth International Conference on , vol., no.,pp.222,229, 8-9 Feb. 2014

[7] Beiranvand, Z.; Patooghy, A; Fazeli, M., "I-LEACH: An efficient routing algorithm to improve performance \& to reduce energy consumption in Wireless Sensor Networks," Information and Knowledge Technology(IKT), 2013 5th Conference on , vol., no., pp.13,18, 28-30 May 2013.

[8] Zhang, J.; Ren, F.; Gao, S.; Yang, H.; Lin, C., "Dynamic Routing for Data Integrity and Delay Differentiated Services in Wireless Sensor Networks," MobileComputing, IEEE Transactions on , vol.PP, no.99, pp.1,1

[9] Al-Anbagi, I; Erol-Kantarci, M.; Mouftah, H.T., "A traffic adaptive inter-cluster head delay control scheme in WSNs," Computers and Communications (ISCC), 2013

[10] Jemal, A; Ben Halima, R., "A QoS-driven Self-Adaptive Architecture for Wireless Sensor Networks," Enabling Technologies: Infrastructure for Collaborative Enterprises (WETICE), 2013 IEEE 22nd International Workshop on , vol., no., pp.125,130, 17-20 June 2013

[11] Dawei Gong, Yuanyuan Yang*, Zhexi Pan, "Energy-efficient clustering in lossy wireless sensor networks", J. Parallel Distrib. Comput. 73 (2013) 1323-1336

[12] Salim EL KHEDIRI, Nejah NASRI, Anne WEI, AbdennaceurKACHOURI," A New Approach for Clustering in Wireless Sensors Networks Based on LEACH", International Workshop on Wireless Networksand Energy Saving Techniques (WNTEST), Procedia Computer Science 32 ( 2014 ) $1180-1185$

[13] Alazzawi, L.K.; Elkateeb, AM.; Ramesh, A, "Scalability Analysis for Wireless Sensor Networks Routing Protocols," Advanced Information Networking andApplications - Workshops, 2008. AINAW 2008. 22nd International Conference on , vol., no., pp.139,144, 25-28 March 2008

[14] Ting Yuan; Shiyong Zhang, "Secure Fault Tolerance in Wireless Sensor Networks," Computer and InformationTechnology Workshops, 2008. CIT Workshops 2008. IEEE 8th International Conference on, vol., no.,pp.477,482, 8-11 July 2008

[15] Tavallaie, O.; Naji, H.R.; Sabaei, M.; Arastouie, N., "Providing QoS guarantee of timeliness in wireless sensor networks with a new routing methodology,"Telecommunications (IST), 2012 SixthInternational Symposium on , vol., no., pp.674,679, 6-8Nov. 2012

[16] Al-Karaki, J.N.; Ul-Mustafa, R.; Kamal, AE., "Data aggregation in wireless sensor networks - exact and approximate algorithms," High Performance Switchingand Routing, 2004. HPSR. 2004 Workshop on , vol., no.,pp.241,245, 2004

[17] O. Younis, S. Fahmy, "HEED: A hybrid, energy-efficient, distributed clustering approach for Ad hoc sensor networks", IEEE Transactions on Mobile Computing, Vol. 3(4), pp. 366-379, Oct.-Dec. 2004.

[18] W. Heinzelman, A. Chandrakasan, and H. Balakrishnan, "Energyefficient routing protocols for wireless microsensor networks", 33 $3^{\text {rd }}$ Hawaii International Conference System Sciences (HICSS), Jan. 2000.

[19] S. Lindesey, C. S. Raghavendra, "PEGASIS: Power-Efficient Gathering in Sensor Information Systems", Proc. Aerospace IEEE Conference, Vol. 3, pp. 3-1125-3-1130, 2002

[20] NehaSaini\&Jasmeet Singh," A Survey: Hierarchal Routing Protocol in Wireless Sensor Networks", Global Journal of Computer Science and Technology: Network, Web \& Security Volume 14 Issue 1 Version 1.0 Year 2014, Online ISSN: 0975-4172 \& Print ISSN: 0975-4350

[21] M. Aslam, N. Javaid, A. Rahim, U. Nazir, A. Bibi, A. Khan "Survey of extended LEACH - based clustering routing protocols for wireless sensor network" IEEE transaction on Antennas and propagation, vol 50 No 5 May 2012.

[22] Sabarish B A, Guru Moorthy S M, Dhivya M A, Sakthi Naveen K, Vaishnavi S "A survey on clustering protocols in Wireless Sensor Networks" International journal of advances in computing and information technology

[23] M.BaniYassein, A.Al-zou'bi, Y.Khaayseh W.Mardini."Improvement of LEACH protocol of Wireless sensor network". Thiemo Voigt, Adam Dunkels, Juan Alonso "Solar-aware Clustering in Wireless Sensor Networks" 